\title{
Rayleigh-Taylor Instability in Magnetized Plasma
}

\author{
G. A. Hoshoudy \\ Department of Applied Mathematics and Computer Science, Faculty of Science, South Valley University, Kena, \\ Egypt \\ Email:g hoshoudy@yahoo.com
}

Received 12 April 2014; revised 8 May 2014; accepted 5 June 2014

Copyright @ 2014 by author and Scientific Research Publishing Inc.

This work is licensed under the Creative Commons Attribution International License (CC BY).

http://creativecommons.org/licenses/by/4.0/

(c) (i) Open Access

\section{Abstract}

The Rayleigh-Taylor instability in stratified plasma has been investigated in the presence of combined effect of horizontal and vertical magnetic field. The linear growth rate has been derived for the case where plasma with exponential density distribution is confined between two rigid planes by solving the linear MHD equations into normal mode. Some special cases have been particularized to explain the roles the variables of the problem play; numerical solutions have been made and some stability diagrams are plotted and discussed. The results show that, the growth rate depends on the horizontal and vertical components of magnetic field and also depends on the parameter $\lambda^{*}=\lambda L_{D} \quad\left(\lambda\right.$ is constant and $L_{D}$ is the density-scale length). The maximum instability happens at $\lambda^{*}=-0.5$ and to get more stability model we select $\lambda^{*}$ such that it is different than $\lambda^{*}=-0.5$. The vertical magnetic field component have a greater effect than the horizontal magnetic field component in the case of large wavelength, while in the case of short wavelength, the horizontal magnetic field components have greater effect than the vertical magnetic field component.

\section{Keywords}

Rayleigh-Taylor Instability, Plasma, Horizontal and Vertical Magnetic Field

\section{Introduction}

It is well-known that, plasma is a hot ionized gas consisting of approximately equal numbers of positively charged ions and negatively charged electrons. The characteristics of plasmas are significantly different from those of ordinary neutral gases so that plasmas are considered a distinct fourth state of matter. There are numer- 
ous everyday uses for plasmas, for example, fluorescent lights and neon signs work because of plasma. Circuit features on micro-processor chips in computers also contain plasma. Even the exhaust that is emitted during rocket launches is actually plasma, not gas. Plasma is also used to manufacture industrial diamonds and superconducting films. Plasma is also a key technology in the development of alternative energy sources. Nuclear fusion, which is plasma based, is one of the most promising candidates for the energy needs of the future when fossil fuels finally run out. One of the important models rises in hydrodynamic plasma called the RayleighTaylor instability (RTI) problem [1] [2]. RTI can occur when dense plasma is supported against the gravity. Studies on RTI show that this instability is troublesome because it obstructs the realization of ICF [3] [4]. In astrophysics, this instability is related to the stellar structure and evolution [5] [6]. Hence, it is important to understand the physical mechanisms that can affect such instability, especially its suppression as well as the details of this instability. Such knowledge will aid our understanding of the origin of white dwarfs and type-Ia supernovas.

Also, it is well-known that, the plasmas are strongly influenced by magnetic fields (where the plasma parameters may vary with the application of magnetic field), where the behavior of plasmas in the presence of a magnetic field is among the oldest problems in plasma physics. It has been central in plasma fusion research since the early experiments on plasma confinement by a magnetic field in the 1950s, and it has remained great interest in plasma fusion studies that use contemporary sophisticated devices [7]. It is also an important problem for many plasma discharges used in processing semiconductor materials where the application of a magnetic field results in enhancement of some desirable features of specific plasma sources [8]. The linear growth rate of RTI has been obtained by Goldston and Rutherford [9] under fixed boundary conditions. The hydromagnetic stability of a magnetized plasma of variable density is of considerable importance in several astrophysical situations, e.g. in theories of sunspot magnetic fields, heating of solar corona and the stability of stellar atmospheres in magnetic fields. Ariel [10] investigated the stability of an inviscid compressible fluid of variable density in the presence of a uniform vertical magnetic field and viscous plasma has been investigated by Bhatia [11]. The instability of stratified plasmas in the presence of horizontal magnetic field of compressible plasmas is studied by Bhimsen [12]. The RTI of a plasma layer in the presence of a horizontal magnetic field is investigated, taking into account the effects of Hall-currents and an arbitrarily large density gradient by Ariel [13]. The effects of horizontal magnetic field, Hall currents and viscosity have been studied on the RTI of an incompressible infinitely conducting stratified plasma by Ahsan and Bhatia [14]. The effect of horizontal magnetic field of Hall currents have been investigated on the RTI of a finitely conducting stratified partially ionized plasma by Aiyub and Bhatia [15]. The instability of stratified plasmas in the presence of horizontal magnetic field of incompressible plasmas with the effect of a transverse velocity shear was studied by Wu et al. [16]. The effect horizontal magnetic field on RTI of a plasma layer in the presence of quantum mechanism was studied by Jintao et al. [17]. The effects of vertical magnetic field in the presence of quantum mechanism on RTI inviscous and viscous plasma were studied by Hoshoudy [18] [19]. The effects of magnetic field gradient on the Rayleigh-Taylor instability (RTI) with continuous magnetic field and density profiles were analytically investigated by Yang et al. [20]. The analytically and numerically investigated stabilization of the linear growth of the RTI from density gradients, magnetic fields, and quantum effects, in an ideal incompressible magnetized plasma was studied by Wang et al. [21]. Because of the great scientific interest in magnetized plasma problem, it is attempted to discuss the RTI problem of a stratified plasma layer in the presence of an variable magnetic field, where this problem corresponds physically (in astrophysics) to the RTI of an equatorial section of a planetary magnetosphere or of a stellar atmosphere where the magnetic field are perpendicular or parallels to gravity. In all the above-mentioned studies the behaviour of growth rates is considered in the presence of a variable magnetic field in $x$ - direction only or in $z$ - direction only. Here, the effect of magnetic field in both $x-$ and $z$ - direction on RTI problem for a finite thickness layer of incompressible plasmas are studied. The dispersion relation is obtained analytically and numerically analyzed.

\section{Linearized Equations}

We consider the strata of incompressible and inviscous plasma as a fluid of electrons and immobile ions. The plasma is immersed in a magnetic field, where the relevant liner perturbation equations may be written as (Refs. [9]-[17])

$$
\rho_{0} \frac{\partial \overrightarrow{\boldsymbol{U}}_{1}}{\partial t}=-\vec{\nabla} p_{1}+\rho_{1} \overrightarrow{\boldsymbol{g}}+\frac{1}{\mu_{0}}\left[\left(\nabla \times B_{0}\right) \times B_{1}+\left(\nabla \times B_{1}\right) \times B_{0}\right],
$$




$$
\begin{aligned}
& \nabla \cdot \overrightarrow{\boldsymbol{U}}_{1}=0 \\
& \frac{\partial \overrightarrow{\boldsymbol{B}}_{1}}{\partial t}=\overrightarrow{\boldsymbol{V}} \times\left(\overrightarrow{\boldsymbol{U}}_{1} \times \overrightarrow{\boldsymbol{B}}_{0}\right) \\
& \frac{\partial \rho_{1}}{\partial t}+\left(\overrightarrow{\boldsymbol{U}}_{1} \cdot \vec{\nabla}\right) \rho_{0}=0
\end{aligned}
$$

Here $\overrightarrow{\boldsymbol{U}}_{1}, p_{1}, p_{1}$ and $\rho_{1}$, are the perturbations in the velocity $\overrightarrow{\boldsymbol{U}}$, pressure $p$, magnetic field $\overrightarrow{\boldsymbol{B}}$, and density $\rho$, respectively. While $\overrightarrow{\boldsymbol{U}}_{1}=\left(u_{x 1}, u_{y 1}, u_{z 1}\right), \overrightarrow{\boldsymbol{B}}_{1}=\left(B_{x 1}, B_{y 1}, B_{z 1}\right), \overrightarrow{\boldsymbol{g}}=(0,0,-g)$ and the fluid is arranged in horizontal strata, then $\rho_{0}$ is a function of the vertical coordinate $z$ only (i.e. $\rho_{0}=\rho_{0}(z)$ ) and $\overrightarrow{\boldsymbol{B}}_{0}=B_{x 0}(z) \overrightarrow{\boldsymbol{e}}_{x}+B_{z 0}(z) \overrightarrow{\boldsymbol{e}}_{z}$. Then the system of Equations (1)-(4) become:

$$
\begin{aligned}
& \rho_{0} \frac{\partial u_{x 1}}{\partial t}=-\frac{\partial p}{\partial x}+\frac{1}{\mu_{0}}\left\{B_{z 1} \frac{\partial B_{x 0}(z)}{\partial z}+B_{0 z}(z)\left(\frac{\partial B_{x 1}}{\partial z}-\frac{\partial B_{z 1}}{\partial x}\right)\right\}, \\
& \rho_{0} \frac{\partial u_{y 1}}{\partial t}=-\frac{\partial p}{\partial y}+\frac{1}{\mu_{0}}\left\{-B_{x 0}(z)\left(\frac{\partial B_{y 1}}{\partial x}-\frac{\partial B_{x 1}}{\partial y}\right)+B_{0 z}(z)\left(\frac{\partial B_{y 1}}{\partial z}-\frac{\partial B_{x 1}}{\partial y}\right)\right\}, \\
& \rho_{0} \frac{\partial u_{z 1}}{\partial t}=-\frac{\partial p}{\partial z}+\frac{1}{\mu_{0}}\left\{-B_{x 1} \frac{\partial B_{x 0}(z)}{\partial z}+B_{x 0}(z)\left(\frac{\partial B_{z 1}}{\partial x}-\frac{\partial B_{x 1}}{\partial z}\right)\right\}-\rho_{1} g, \\
& \frac{\partial u_{x 1}}{\partial x}+\frac{\partial u_{y 1}}{\partial y}+\frac{\partial u_{z 1}}{\partial z}=0, \\
& \frac{\partial \overrightarrow{B_{1}}}{\partial t}=\frac{\partial}{\partial t}\left(B_{x 1}, B_{y 1}, B_{z 1}\right)=\left\{\begin{array}{l}
{\left[-B_{x 0}(z) \frac{\partial u_{y 1}}{\partial y}+\frac{\partial}{\partial z}\left(B_{z 0}(z) u_{x 1}-B_{x 0}(z) u_{z 1}\right)\right],} \\
\left.[z) \frac{\partial u_{y 1}}{\partial x}-\frac{\partial}{\partial z}\left(B_{z 0}(z) u_{y 1}\right)\right], \\
{\left[\frac{\partial}{\partial x}\left(B_{x 0}(z) u_{z 1}-B_{z 0}(z) u_{x 1}\right)-B_{z 0}(z) \frac{\partial u_{y 1}}{\partial y}\right] .}
\end{array}\right\}, \\
& \frac{\partial \rho_{1}}{\partial t}+u_{z 1} \frac{\mathrm{d} \rho_{0}(z)}{\mathrm{d} z}=0 .
\end{aligned}
$$

If we assume that the perturbation in any physical quantity takes the form:

$$
\psi_{1}(x, y, z, t)=\psi_{1}(z) \exp \left\{i\left(k_{x} x+k_{y} y-\omega t\right)\right\},
$$

where $k_{x}$ and $k_{y}$ are horizontal components of the wave-number vector $\boldsymbol{k}$ such that $\boldsymbol{k}^{2}=k_{x}^{2}+k_{y}^{2}$ and $\omega$ (may be complex $\left.\left(\omega=\omega_{r}+i \gamma\right)\right)$ is the frequency of perturbations or the rate at which the system departs from equilibrium. Using the Expression (11) in the system of Equations (5) - (10), we have:

$$
\begin{aligned}
& -i \rho_{0} \omega u_{x 1}=-i k_{x} p_{1}+\frac{1}{\mu_{0}}\left\{B_{z 1} \frac{\partial B_{x 0}(z)}{\partial z}+B_{z 0}(z)\left[\frac{\partial B_{x 1}(z)}{\partial z}-i k_{x} B_{z 1}\right]\right\}, \\
& -i \rho_{0} \omega u_{y 1}=-i k_{y} p_{1}+\frac{1}{\mu_{0}}\left\{i B_{x 0}(z)\left[k_{x} B_{y 1}-k_{y} B_{x 1}\right]+B_{z 0}(z)\left[\frac{\partial B_{y 1}(z)}{\partial z}-i k_{y} B_{z 1}\right]\right\}, \\
& -i \rho_{0} \omega u_{z 1}=-\frac{\partial p_{1}}{\partial z}-\rho_{1} g+\frac{1}{\mu_{0}}\left\{-B_{x 1} \frac{\partial B_{x 0}(z)}{\partial z}+B_{x 0}(z)\left[i k_{x} B_{z 1}-\frac{\partial B_{x 1}(z)}{\partial z}\right]\right\}, \\
& i k_{x} u_{x 1}+i k_{y} u_{y 1}+\frac{\partial u_{z 1}}{\partial z}=0
\end{aligned}
$$




$$
\begin{aligned}
-i \omega\left\{B_{x 1}, B_{y 1}, B_{z 1}\right\}=\left\{\begin{array}{l}
{\left[-i k_{y} B_{x 0}(z) u_{y 1}+\frac{\partial}{\partial z}\left(B_{z 0}(z) u_{x 1}-B_{x 0}(z) u_{z 1}\right)\right],} \\
{\left[i k_{x} B_{x 0}(z) u_{y 1}-\frac{\partial}{\partial z}\left(B_{z 0}(z) u_{y 1}\right)\right],} \\
{\left[i k_{x}\left(B_{x 0}(z) u_{z 1}-B_{z 0}(z) u_{x 1}\right)-i k_{y} B_{z 0}(z) u_{y 1}\right] .}
\end{array}\right\}, \\
-i \omega \rho_{1}+u_{z 1} \frac{\mathrm{d} \rho_{0}}{\mathrm{~d} z}=0
\end{aligned}
$$

Eliminating some variables from the system of Equations (12) - (17) we have:

$$
\begin{aligned}
& \frac{B_{z 0}^{2}(z)}{\mu_{0}} \frac{\mathrm{d}^{4} u_{z 1}}{\mathrm{~d} z^{4}}+\frac{1}{\mu_{0}}\left\{4 B_{0 z}(z)\left(\frac{\mathrm{d} B_{0}(z)}{\mathrm{d} z}\right)+2 i k_{x} B_{x 0}(z) B_{z 0}(z)\right\} \frac{\mathrm{d}^{3} u_{z 1}}{\mathrm{~d} z^{3}}+\left\{\rho_{0} \omega^{2}+A\right\} \frac{\mathrm{d}^{2} u_{z 1}}{\mathrm{~d} z^{2}} \\
& +\left\{\omega^{2}\left(\frac{\mathrm{d} \rho_{0}}{\mathrm{~d} z}\right)+B\right\} \frac{\mathrm{d} u_{z 1}}{\mathrm{~d} z}-k^{2}\left\{\rho_{0} \omega^{2}-C+g\left(\frac{\mathrm{d} \rho_{0}}{\mathrm{~d} z}\right)\right\} u_{z 1}=0,
\end{aligned}
$$

where,

$$
\begin{aligned}
& A=\frac{1}{\mu_{0}}\left\{-k_{x}^{2} B_{x 0}^{2}(z)+3 B_{z 0}(z) \frac{\mathrm{d}^{2} B_{z 0}(z)}{\mathrm{d} z^{2}}+2\left(\frac{\mathrm{d} B_{z 0}(z)}{\mathrm{d} z}\right)^{2}-k^{2} B_{z 0}^{2}(z)+3 i k_{x}\left[B_{x 0}(z) \frac{\mathrm{d} B_{z 0}(z)}{\mathrm{d} z}+B_{z 0}(z) \frac{\mathrm{d} B_{x 0}(z)}{\mathrm{d} z}\right]\right\} \\
& B=\frac{1}{\mu_{0}}\left\{\begin{array}{l}
\left.\left.-2 k_{x}^{2} B_{x 0}(z) \frac{\mathrm{d} B_{x 0}(z)}{\mathrm{d} z}+B_{z 0}(z) \frac{\mathrm{d}^{3} B_{z 0}(z)}{\mathrm{d} z^{3}}+\frac{\mathrm{d} B_{z 0}(z)}{\mathrm{d} z} \frac{\mathrm{d}^{2} B_{z 0}(z)}{\mathrm{d} z^{2}}-2 k^{2} B_{z 0}(z) \frac{\mathrm{d} B_{z 0}(z)}{\mathrm{d} z}\right]+i k_{x}\left[2 B_{z 0}(z) \frac{\mathrm{d}^{2} B_{x 0}(z)}{\mathrm{d} z^{2}}-2 k^{2} B_{x 0}(z) B_{z 0}(z)+2 \frac{\mathrm{d} B_{x 0}(z)}{\mathrm{d} z} \frac{\mathrm{d} B_{z 0}(z)}{\mathrm{d} z}+\frac{\mathrm{d} B_{x 0}(z)}{\mathrm{d} z} \frac{\mathrm{d}^{2} B_{z 0}(z)}{\mathrm{d} z^{2}}\right]\right\} \\
c=\frac{1}{\mu_{0}}\left\{k_{x}^{2} B_{x 0}^{2}(z)+i k_{x}\left[\frac{1}{k^{2}}\left(B_{z 0}(z) \frac{\mathrm{d}^{3} B_{z 0}(z)}{\mathrm{d} z^{3}}+\frac{\mathrm{d} B_{z 0}(z)}{\mathrm{d} z} \frac{\mathrm{d}^{3} B_{x 0}(z)}{\mathrm{d} z^{3}}\right)-B_{x 0}(z) \frac{\mathrm{d} B_{z 0}(z)}{\mathrm{d} z}-B_{z 0}(z) \frac{\mathrm{d} B_{x 0}(z)}{\mathrm{d} z}\right]\right\} .
\end{array}\right.
\end{aligned}
$$

\section{A Continuously Stratified Plasma Layer}

In this section we consider the case of incompressible continuously stratified plasma layer of thickness $h$ units confined between two rigid boundaries, in which the density and magnetic field distribution are given, respectively, by $\rho_{0}(z)=\rho_{0}(0) \exp \left(z / L_{D}\right), B_{x 0}(z)=B_{x 0}(0) \exp \left(z / 2 L_{D}\right)$ and $B_{z 0}(z)=B_{z 0}(0) \exp \left(z / 2 L_{D}\right)$ where $\rho_{0}(0), B_{x 0}(0)$ and $B_{z 0}(0)$ and $L_{D}$ (the density-scale length) are constants, then Equation (18) takes the form:

$$
\begin{aligned}
& v_{f_{z}}^{2} \frac{\mathrm{d}^{4} u_{z 1}}{\mathrm{~d} z^{4}}+\frac{2}{L_{D}}\left\{v_{f_{z}}^{2}+i k_{x} L_{D} v_{f_{z}} v_{f_{x}}\right\} \frac{\mathrm{d}^{3} u_{z 1}}{\mathrm{~d} z^{3}}+\left\{\omega^{2}-k_{x}^{2} v_{f_{x}}^{2}+v_{f_{z}}^{2}\left(\frac{5}{4 L_{D}^{2}}-k^{2}\right)+\frac{3 i k_{x} v_{f_{z}} v_{f_{x}}}{L_{D}}\right\} \frac{\mathrm{d}^{2} u_{z 1}}{\mathrm{~d} z^{2}} \\
& +\frac{1}{L_{D}}\left\{\omega^{2}-k_{x}^{2} v_{f_{x}}^{2}+v_{f_{z}}^{2}\left(\frac{1}{4 L_{D}^{2}}-k^{2}\right)+i k_{x} L_{D} v_{f_{z}} v_{f_{x}}\left(\frac{5}{4 L_{D}^{2}}-2 k^{2}\right)\right\} \frac{\mathrm{d} u_{z 1}}{\mathrm{~d} z} \\
& -k^{2}\left\{\omega^{2}+\frac{g}{L_{D}}-k_{x}^{2} v_{f_{x}}^{2}-i k_{x} v_{f_{z}} v_{f_{x}}\left(\frac{1}{4 k^{2} L_{D}^{3}}-\frac{1}{L_{D}}\right)\right\} u_{z 1}=0,
\end{aligned}
$$

where $v_{f_{x}}^{2}=\frac{B_{x 0}^{2}(0)}{\mu_{0} \rho_{0}(0)}$ and $v_{f_{z}}^{2}=\frac{B_{z 0}^{2}(0)}{\mu_{0} \rho_{0}(0)}$ are Alfvén velocity. Now, if we choose $u_{z 1}$ in the form 
$u_{z 1}=\sin \left(\frac{n \pi}{h} z\right) \exp (\lambda z)$ and by substituting in Equation (26), we will have an equation in both $\sin \left(\frac{n \pi}{h} z\right)$ and $\cos \left(\frac{n \pi}{h} z\right)$. Then coefficients both $\sin \left(\frac{n \pi}{h} z\right)$ and $\cos \left(\frac{n \pi}{h} z\right)$, respectively, are given by:

$$
\begin{aligned}
& v_{f_{z}}^{2}\left\{\lambda^{4}+\left(\frac{n \pi}{h}\right)^{4}-6 \lambda^{2}\left(\frac{n \pi}{h}\right)^{2}\right\}+\frac{2 \lambda}{L_{D}}\left\{v_{f_{z}}^{2}+i k_{x} L_{D} v_{f_{z}} v_{f_{x}}\right\}\left\{\lambda^{2}-3\left(\frac{n \pi}{h}\right)^{2}\right\} \\
& +\left\{\omega^{2}-k_{x}^{2} v_{f_{x}}^{2}+v_{f_{z}}^{2}\left(\frac{5}{4 L_{D}^{2}}-k^{2}\right)+\frac{3 i k_{x} v_{f_{z}} v_{f_{x}}}{L_{D}}\right\}\left\{\lambda^{2}-\left(\frac{n \pi}{h}\right)^{2}\right\} \\
& +\frac{\lambda}{L_{D}}\left\{\omega^{2}-k_{x}^{2} v_{f_{x}}^{2}+v_{f_{z}}^{2}\left(\frac{1}{4 L_{D}^{2}}-k^{2}\right)+i k_{x} L_{D} v_{f_{z}} v_{f_{x}}\left(\frac{5}{4 L_{D}^{2}}-2 k^{2}\right)\right\} \\
& -k^{2}\left\{\omega^{2}+\frac{g}{L_{D}}-k_{x}^{2} v_{f_{x}}^{2}-i k_{x} v_{f_{z}} v_{f_{x}}\left(\frac{1}{4 k^{2} L_{D}^{3}}-\frac{1}{L_{D}}\right)\right\}=0, \\
& 4 \lambda v_{f_{z}}^{2}\left\{\lambda^{2}-\left(\frac{n \pi}{h}\right)^{2}\right\}+2 \lambda\left\{\omega^{2}-k_{x}^{2} v_{f_{x}}^{2}+v_{f_{z}}^{2}\left(\frac{5}{4 L_{D}^{2}}-k^{2}\right)\right\}+\frac{2 v_{f_{z}}^{2}}{L_{D}}\left\{3 \lambda^{2}-\left(\frac{n \pi}{h}\right)^{2}\right\} \\
& +\frac{1}{L_{D}}\left\{\omega^{2}-k_{x}^{2} v_{f_{x}}^{2}+v_{f_{z}}^{2}\left(\frac{1}{4 L_{D}^{2}}-k^{2}\right)\right\}+i k_{x} v_{f_{z}} v_{f_{x}}\left\{\frac{6 \lambda}{L_{D}}+2\left(3 \lambda^{2}-\left(\frac{n \pi}{h}\right)^{2}\right)+\left(\frac{5}{4 L_{D}^{2}}-2 k^{2}\right)\right\}=0 .
\end{aligned}
$$

Now, we define the dimensionless quantities:

$$
\begin{aligned}
& \omega^{* 2}=\frac{\omega^{2}}{\omega_{p e}^{2}}, \quad \omega_{f_{x}}^{* 2}=\frac{v_{f_{x}}^{2}}{\omega_{p e}^{2} L_{D}^{2}}, \quad \omega_{f_{z}}^{* 2}=\frac{v_{f_{z}}^{2}}{\omega_{p e}^{2} L_{D}^{2}}, \quad \lambda^{* 2}=\lambda^{2} L_{D}^{2}, \quad h^{* 2}=\frac{h^{2}}{L_{D}^{2}}, \\
& k^{* 2}=k^{2} L_{D}^{2}, \quad g^{*}=\frac{g}{\omega_{p e}^{2} L_{D}}, \quad \omega_{p e}=\left(\frac{\rho_{0} \mathrm{e}^{2}}{m_{e}^{2}}\right)^{1 / 2} .
\end{aligned}
$$

Then Equations (21) and (22), respectively, take the form:

$$
\begin{aligned}
& \left\{\lambda^{* 2}+\lambda^{*}-\left(\frac{n \pi}{h^{*}}\right)^{2}-k^{* 2}\right\}\left\{\omega^{* 2}-k_{x}^{* 2} \omega_{f_{x}}^{* 2}\right\} \\
& +\left\{\lambda^{* 4}+\left(\frac{n \pi}{h^{*}}\right)^{4}-6 \lambda^{* 2}\left(\frac{n \pi}{h^{*}}\right)^{2}+2 \lambda^{*}\left(\lambda^{* 2}-3\left(\frac{n \pi}{h^{*}}\right)^{2}\right)+\left(\frac{5}{4}-k^{* 2}\right)\left(\lambda^{* 2}-\left(\frac{n \pi}{h^{*}}\right)^{2}\right)+\lambda^{*}\left(\frac{1}{4}-k^{* 2}\right)\right\} \omega_{f_{z}}^{* 2} \\
& +\left\{2 \lambda^{*}\left(\lambda^{* 2}-3\left(\frac{n \pi}{h^{*}}\right)^{2}\right)+3\left(\lambda^{* 2}-\left(\frac{n \pi}{h^{*}}\right)^{2}\right)+\lambda^{*}\left(\frac{5}{4}-2 k^{* 2}\right)+\left(\frac{1}{4}-k^{* 2}\right)\right\} i k_{x}^{*} \omega_{f_{x}}^{*} \omega_{f_{z}}^{*}-k^{* 2} g^{*}=0 . \\
& \left\{2 \lambda^{*}+1\right\}\left\{\omega^{* 2}-k_{x}^{* 2} \omega_{f_{x}}^{* 2}+\left[2 \lambda^{* 2}+2 \lambda^{*}+\frac{1}{4}-2\left(\frac{n \pi}{h^{* 2}}\right)^{2}-k^{* 2}\right] \omega_{f_{z}}^{* 2}\right\} \\
& +\left\{6 \lambda^{* 2}+6 \lambda^{*}-2\left(\frac{n \pi}{h^{* 2}}\right)^{2}+\left(\frac{5}{4}-2 k^{* 2}\right)\right\} i k_{x}^{*} \omega_{f_{x}}^{*} \omega_{f_{z}}^{*}=0 .
\end{aligned}
$$
by:

Now, we put $\omega^{*}=\omega_{r}^{*}+i \gamma$ and for $\omega_{r}^{*}=0$ (stable oscillations), then Equations (24) and (25) may be given 


$$
\begin{aligned}
& \left\{\lambda^{* 2}+\lambda^{*}-\left(k^{* 2}+\left(\frac{n \pi}{h^{*}}\right)^{2}\right)\right\}\left\{-\gamma^{2}-k_{x}^{* 2} \omega_{f_{x}}^{* 2}\right\} \\
& +\left\{\lambda^{* 4}+\left(\frac{n \pi}{h^{*}}\right)^{4}-6 \lambda^{* 2}\left(\frac{n \pi}{h^{*}}\right)^{2}+2 \lambda^{*}\left(\lambda^{* 2}-3\left(\frac{n \pi}{h^{*}}\right)^{2}\right)+\left(\frac{5}{4}-k^{* 2}\right)\left(\lambda^{* 2}-\left(\frac{n \pi}{h^{*}}\right)^{2}\right)+\lambda^{*}\left(\frac{1}{4}-k^{* 2}\right)\right\} \omega_{f_{z}}^{* 2} \\
& +\left\{2 \lambda^{*}\left(\lambda^{* 2}-3\left(\frac{n \pi}{h^{*}}\right)^{2}\right)+3\left(\lambda^{* 2}-\left(\frac{n \pi}{h^{*}}\right)^{2}\right)+\lambda^{*}\left(\frac{5}{4}-2 k^{* 2}\right)+\left(\frac{1}{4}-k^{* 2}\right)\right\} i k_{x}^{*} \omega_{f_{x}}^{*} \omega_{f_{z}}^{*}-k^{* 2} g^{*}=0, \\
& \left\{2 \lambda^{*}+1\right\}\left\{\left\{-\gamma^{2}-k_{x}^{* 2} \omega_{f_{x}}^{* 2}\right\}+\left\{2 \lambda^{* 2}+2 \lambda^{*}+\frac{1}{4}-2\left(\frac{n \pi}{h^{*}}\right)^{2}-k^{* 2}\right\}\right\} \omega_{f_{z}}^{* 2} \\
& +\left\{6 \lambda^{* 2}+6 \lambda^{*}-2\left(\frac{n \pi}{h^{*}}\right)^{2}+\left(\frac{5}{4}-2 k^{* 2}\right)\right\} i k_{x}^{*} \omega_{f_{x}}^{*} \omega_{f_{z}}^{*}=0 .
\end{aligned}
$$

To discuss the role of parameter's problem we consider the next special cases from Equations (26) and (27).

(i) In the case of $\omega_{f_{x}}^{* 2}=\omega_{f_{z}}^{* 2}=0$.

From Equation (27) we get $\lambda^{*}=-0.5$, and substituting in Equation (26) we find that the square normalized growth rate given by:

$$
\gamma_{\text {Goldston and Rutherford }}^{2}=\frac{k^{* 2} g^{*}}{\frac{1}{4}+\left(\frac{n \pi}{h^{*}}\right)^{2}+k^{* 2}},
$$

This case is considered by Goldston and Rutherford (see Ref. [9]), which represents an exponentially growing perturbation (instability case).

(ii) In the case of $\omega_{f_{z}}^{* 2}=0, \omega_{f_{x}}^{* 2} \neq 0$.

A second time, from Equation (27) we get $\lambda^{*}=-0.5$, and substituting in Equation (26), then the square normalized growth rate given by:

$$
\gamma_{\text {horizont magnetic field }}^{2}=\frac{k^{* 2} g^{*}}{\frac{1}{4}+\left(\frac{n \pi}{h^{*}}\right)^{2}+k^{* 2}}-k_{x}^{* 2} \omega_{f_{x}}^{* 2} .
$$

This case studied in Refs. ([16] [17]). It is clarified that, the horizontal magnetic field has stabilizing effect on RTI problem. This influence is obvious from Equations (28) and (29), where,

$\gamma_{\text {horizont magnetic field }}^{2}<\gamma_{\text {Goldston and Rutherford }}^{2}$. Also, one can see that the square normalized growth rate decreases as $\omega_{f_{x}}^{*^{2}}$ increases and the system arrives to complete stability case at $k_{c}^{* 2}=\frac{\frac{1}{4}+\left(\frac{n \pi}{h^{*}}\right)^{2}}{g^{*}-k_{x}^{* 2} \omega_{f_{x}}^{* 2}} k_{x}^{* 2} \omega_{f_{x}}^{* 2}$. Now, if we use the relation $k^{* 2}=k_{x}^{* 2}+k_{y}^{* 2} \Rightarrow k_{x}^{* 2}=k^{* 2}-k_{y}^{* 2}$ under the condition $k_{y}^{* 2}<k^{* 2} \Rightarrow k_{y}^{* 2}<k^{* 2} \Rightarrow k_{y}^{* 2}=\bar{C} k^{* 2}, \bar{C}<1$ then $k_{x}^{* 2}=k^{* 2}-\bar{C} k^{2}=k^{* 2}(1-\bar{C})=C k^{* 2}, C<1$. Then Equation (29) becomes:

$$
\gamma_{\text {horizont magnetic field }}^{2}=\frac{k^{* 2} g^{*}}{\frac{1}{4}+\left(\frac{n \pi}{h^{*}}\right)^{2}+k^{* 2}}-C k^{* 2} \omega_{f_{x}}^{* 2} .
$$

At $\gamma_{\text {horizont magnetic field }}^{2}=0$, the critical point becomes $k_{c}^{* 2}=\frac{g^{*}}{C \omega_{f_{x}}^{* 2}}-\left\{\frac{1}{4}+\left(\frac{n \pi}{h^{*}}\right)^{2}\right\}$.

This implies that the complete stability happens at $\omega_{f_{x}}^{* 2}<\frac{g^{*}}{C\left\{\left(\frac{n \pi}{h^{*}}\right)^{2}+\frac{1}{4}\right\}}$. 
Also, if the perturbation in the Equation (11) in the form $f_{1}(x, y, z, t)=f(z) \exp (i(k x-\omega t))$. Then $C=1$ and the square normalized growth rate becomes $\gamma_{\text {horizont magnetic field }}^{2}=\frac{k^{* 2} g^{*}}{\frac{1}{4}+\left(\frac{n \pi}{h^{*}}\right)^{2}+k^{* 2}}-k^{* 2} \omega_{f_{x}}^{* 2}$, and the critical point of stability becomes $k_{c}^{* 2}=\frac{g^{*}}{\omega_{f_{x}}^{* 2}}-\left\{\frac{1}{4}+\left(\frac{n \pi}{h^{*}}\right)^{2}\right\}$, this results correspond with Ref. [17].

(iii) In the case of $\omega_{f_{x}}^{* 2}=0, \omega_{f_{z}}^{* 2} \neq 0$. Again, from Equation (27) we get $\lambda^{*}=-0.5$, and substituting in Equation (26), the square normalized growth rate given by:

$$
\gamma_{\text {vertical magnetic field }}^{2}=\frac{g^{*} k^{* 2}}{\frac{1}{4}+\left(\frac{n \pi}{h^{*}}\right)^{2}+k^{* 2}}-\frac{\left\{\left(\frac{n \pi}{h^{*}}\right)^{2}+\frac{1}{4}\right\}\left\{\left(\frac{n \pi}{h^{*}}\right)^{2}+k^{* 2}\right\} \omega_{f_{z}}^{* 2}}{\frac{1}{4}+\left(\frac{n \pi}{h^{*}}\right)^{2}+k^{* 2}} .
$$

Now, comparing between Equations (28) and (31), someone can observe that, the stabilizing role for the vertical magnetic field on the considerable system, where $\gamma_{\text {vertical magnetic field }}^{2}<\gamma_{\text {Goldston and Rutherford }}^{2}$ and the system arrives to complete stability case at $k_{c}^{* 2}=\frac{\left(\frac{n \pi}{h^{*}}\right)^{2}}{\left\{\left(\frac{n \pi}{h^{*}}\right)^{2}+\frac{1}{4}\right\}^{*} \omega_{f_{z}}^{* 2}}$. This indicates that, in the presence of $\omega_{f_{z}}^{*}$, the stability role stratifies under the condition $\omega_{f_{z}}^{* 2}<\frac{g^{*}}{\left(\frac{n \pi}{h^{*}}\right)^{2}+\frac{1}{4}}$.

(iv) For the general case $\left(\omega_{f_{z}}^{* 2} \neq 0, \omega_{f_{x}}^{* 2} \neq 0\right)$, if we can eliminate the term $i k_{x}^{*} \omega_{f_{x}}^{*} \omega_{f_{z}}^{*}$ between Equations (26) and (27). Then the square normalized growth rate given in the from

$$
\begin{aligned}
& \gamma^{2}= \\
& \left\{\left\{\lambda^{* 2}+\lambda^{*}-k^{* 2}-\left(\frac{n \pi}{h^{*}}\right)^{2}\right\}\left\{6 \lambda^{* 2}+6 \lambda^{*}-2 k^{* 2}-2\left(\frac{n \pi}{h^{* 2}}\right)^{2}+\frac{5}{4}\right\}-\left\{2 \lambda^{*}+1\right\}\left\{2 \lambda^{*}\left(\lambda^{* 2}-3\left(\frac{n \pi}{h^{*}}\right)^{2}\right)+3\left(\lambda^{* 2}-\left(\frac{n \pi}{h^{*}}\right)^{2}\right)+\lambda^{*}\left(\frac{5}{4}-2 k^{* 2}\right)+\frac{1}{4 k^{* 2}}-1\right\}\right\} \\
& \times\left\{\{ 6 \lambda ^ { * 2 } + 6 \lambda ^ { * } - 2 k ^ { * 2 } - 2 ( \frac { n \pi } { h ^ { * 2 } } ) ^ { 2 } + \frac { 5 } { 4 } \} \left\{\left\{\lambda^{* 2}+\lambda^{*}-k^{* 2}-\left(\frac{n \pi}{h^{*}}\right)^{2}\right\}\left\{-k_{x}^{* 2} \omega_{f_{x}^{* 2}}^{*}\right\}\right.\right. \\
& \left.+\left\{\lambda^{* 4}+\left(\frac{n \pi}{h^{*}}\right)^{4}-6 \lambda^{* 2}\left(\frac{n \pi}{h^{*}}\right)^{2}+\lambda^{*}\left(2 \lambda^{* 2}-k^{* 2}-6\left(\frac{n \pi}{h^{*}}\right)^{2}+\frac{1}{4}\right)+\left(\frac{5}{4}-k^{* 2}\right)\left(\lambda^{* 2}-\left(\frac{n \pi}{h^{*}}\right)^{2}\right)\right\} \omega_{f_{2}}^{* 2}-k^{* 2} g^{*}\right\} \\
& \quad \times\left\{-\left\{2 \lambda^{*}+1\right\}\left\{\left\{-k_{x}^{* 2} \omega_{f_{x}^{* 2}}^{* 2}+\left[2 \lambda^{* 2}+2 \lambda^{*}-k^{* 2}+\frac{1}{4}-2\left(\frac{n \pi}{h^{* 2}}\right)^{2}\right] \omega_{f_{2}}^{* 2}\right\}\left\{\lambda^{* 3}+3 \lambda^{* 2}-6 \lambda^{*}\left(\frac{n \pi}{h^{*}}\right)^{2}-3\left(\frac{n \pi}{h^{*}}\right)^{2}+\lambda^{*}\left(\frac{5}{4}-2 k^{* 2}\right)+\frac{1}{4 k^{* 2}}-1\right\}\right\} .\right.
\end{aligned}
$$

In the general case, if we wish to look into the effect of both horizontal and vertical magnetic field together on the instability of the considered system, Equation(32) is to be numerically solved, where $\gamma^{2}$ is function in the dimensionless quantities of horizontal $\left(\omega_{f_{x}}^{*}\right)$ and vertical $\left(\omega_{f_{z}}^{*}\right)$ components of the magnetic field, the wave number $k^{*}$ and $\lambda^{*}=\lambda L_{D}$, where $\lambda$ is constant and $L_{D}$ is the density-scale length. In these figures $k_{x}^{* 2}=C k^{* 2}, C<1(C=0.2), h^{*}=1, n=1$ and $g^{*} \cong 10$.

The role of $\lambda^{*}$ as a function horizontal $k^{*}$ in the presence of both horizontal and vertical magnetic field components $\left(\omega_{f_{x}}^{*}=\omega_{f_{z}}^{*}=0.25\right)$ is plotted in Figure 1 and Figure 2, where the square normalized growth rate 


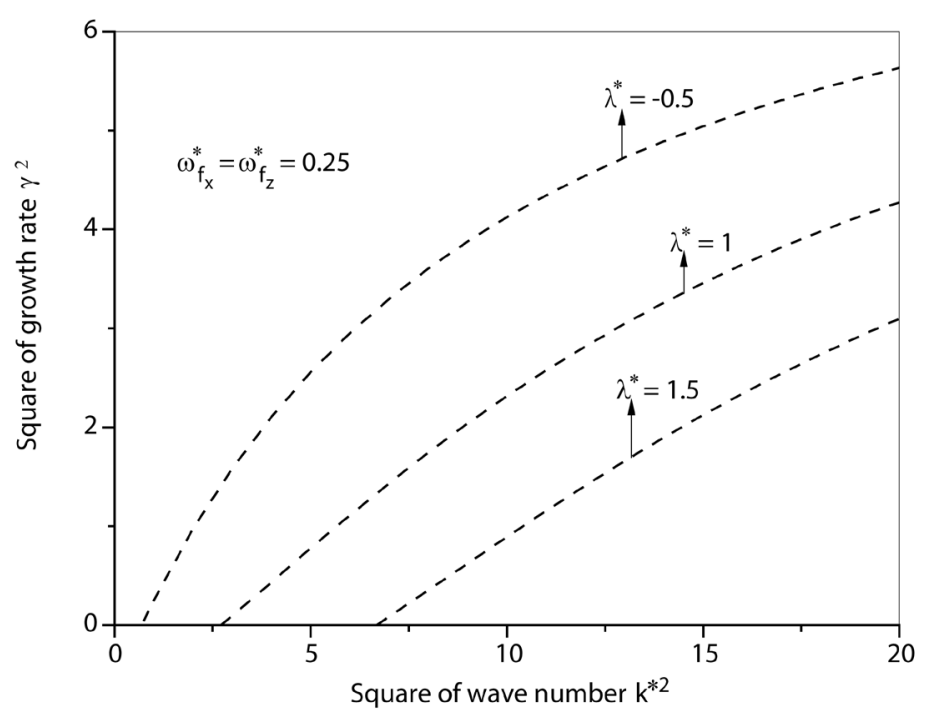

(a)

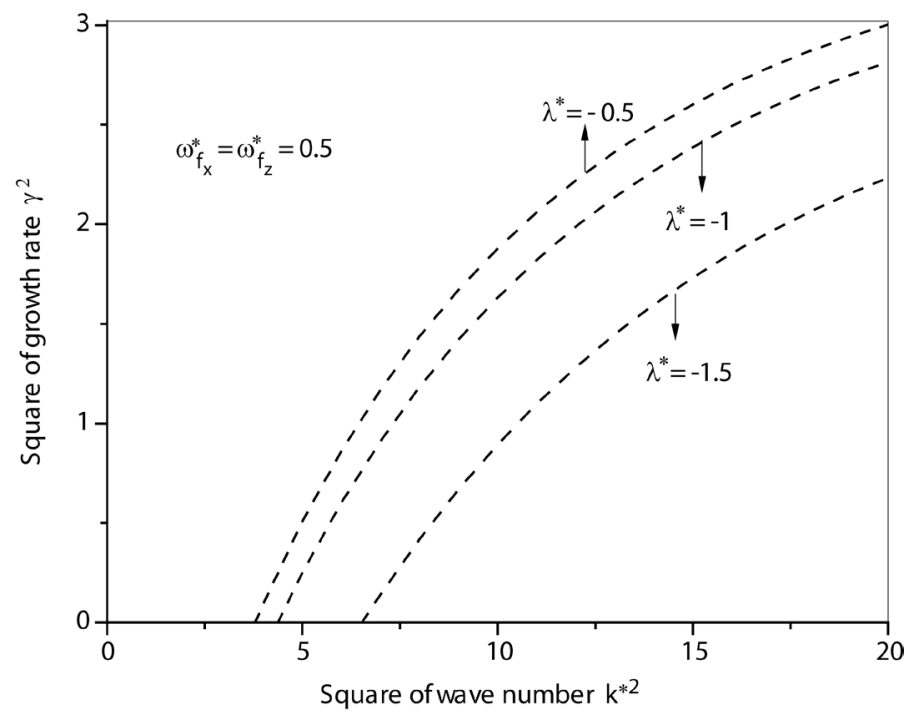

(b)

Figure 1. The square normalized growth rate $\left(\gamma^{2}\right)$ against the square normalized wave number $k^{* 2}$ at $\left(\omega_{f_{x}}^{*}=\omega_{f_{z}}^{*}=0.25\right) \quad$ (a) at $\lambda^{*}=-0.5,1,1.5$ (b) at $\lambda^{*}=-0.5,-1,-1.5$.

$\gamma^{2}$ is plotted against the square normalized wave number $k^{* 2}\left(0 \leq k^{* 2} \leq 20\right)$. Figure 1 (a) shows the role of $\lambda^{*}(\geq-0.5)$, for example $\lambda^{*}=-0.5,1,1.5$. One can see that $\gamma^{2}$ decrease with increasing of $\lambda^{*}$. While Figure (b) shows the role of $\lambda^{*}(\leq-0.5)$ or $\lambda^{*}=-0.5,-1,-1.5$, where $\gamma^{2}$ decrease with decreasing of $\lambda^{*}$. These implies that the maximum instability in the presence of both vertical and horizontal magnetic field happens at $\lambda^{*}=-0.5$. The same phenomenon hold in Figure 2(a) and Figure 2(b) at $\left(\omega_{f_{x}}^{*}=\omega_{f_{z}}^{*}=0.5\right)$. In this case, if we put $\lambda^{*}=-0.5$ in Equation (32), then the maximum square normalized growth rate $\gamma^{2}$ is:

$$
\gamma_{\max }^{2}=\frac{k^{* 2} g^{*}}{\frac{1}{4}+k^{* 2}+\left(\frac{n \pi}{h^{*}}\right)^{2}}-\left\{\frac{\left[\left(\frac{n \pi}{h^{*}}\right)^{4}+\frac{1}{4}\left(\frac{n \pi}{h^{*}}\right)^{2}+\frac{1}{4} k^{* 2}+\left(\frac{n \pi}{h^{*}}\right)^{2} k^{* 2}\right] \omega_{f_{z}}^{* 2}}{\frac{1}{4}+k^{* 2}+\left(\frac{n \pi}{h^{*}}\right)^{2}}+k_{x}^{* 2} \omega_{f_{x}}^{* 2}\right\} .
$$




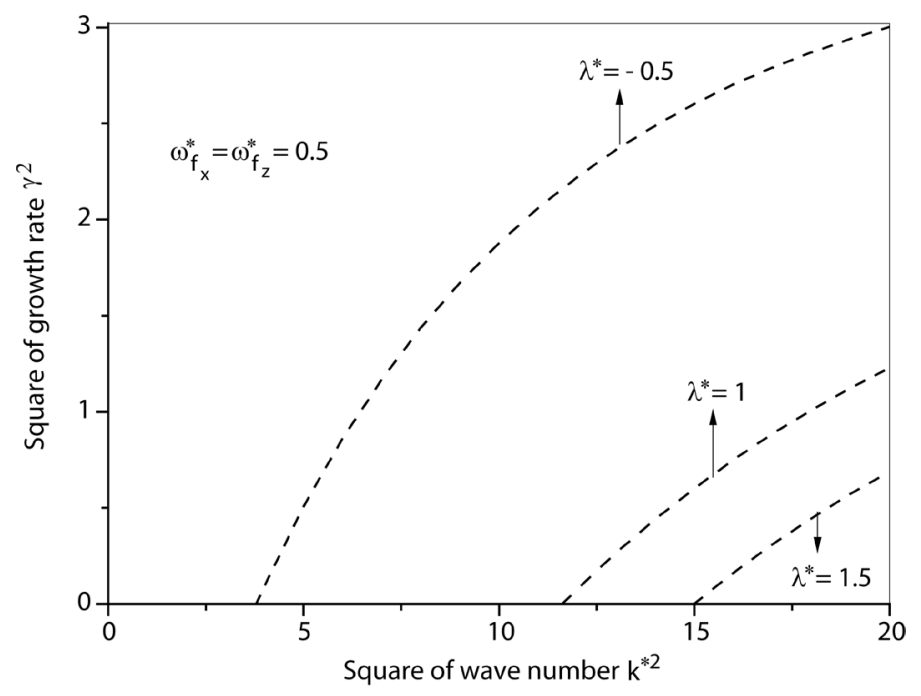

(a)

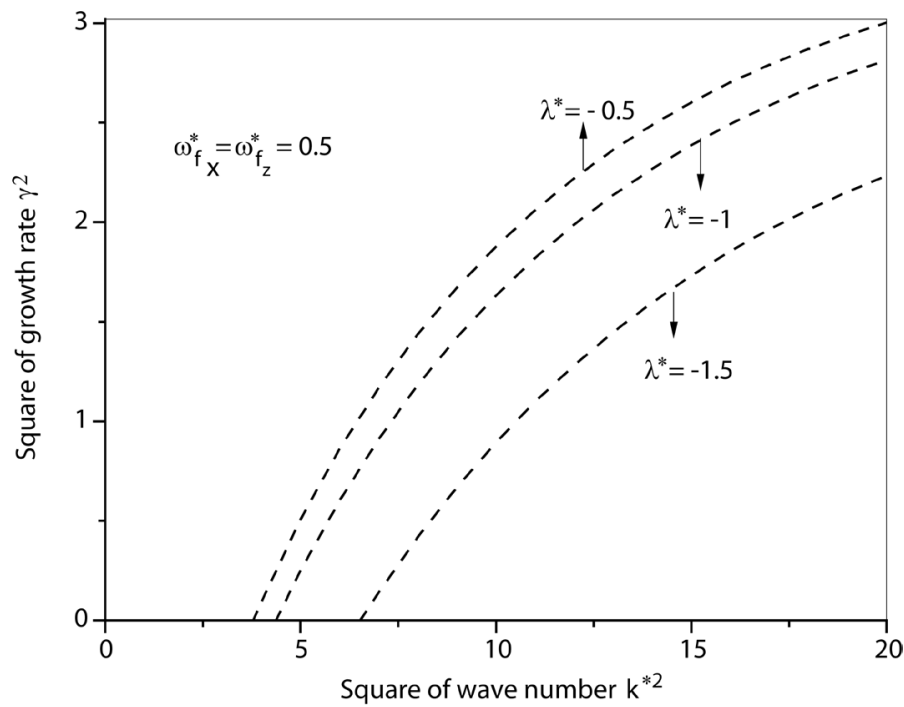

(b)

Figure 2. The same as Figure 1 but at $\left(\omega_{f_{x}}^{*}=\omega_{f_{z}}^{*}=0.5\right)$.

From Equation (33) it can see that, the maximum square normalized growth rate $\gamma^{2}$ (in the presence of both horizontal and vertical magnetic field components) given as we add the second term in Equations (29) and (31) to Equation (28). Figure 3 shows the role of both horizontal and vertical components' magnetic field $\omega_{f_{x}}^{*}=\omega_{f_{z}}^{*}=0.6$ on the considered system at $\lambda^{*}=-0.5$, where the square normalized growth rate $\gamma^{2}$ is plotted against the square normalized wave number $k^{* 2}$. One can see that, the magnitudes of $\gamma^{2}$ in the presence of these parameters $\left(\omega_{f_{x}}^{*}, \omega_{f_{z}}^{*}\right)$ or either them is less than their magnitudes in the Goldston and Rutherford case, which means that these factors have a stabilizing effect on the considered system. Also, it can seen that, no mode of maximum instability exists when $\left(\omega_{f_{x}}^{*}=0\right.$ or $\left.\omega_{f_{z}}^{*}=0\right)$ as the square normalized growth rate $\gamma^{2}$ usually increases by increase with the square normalized wave number values. While, in the presence of quantum term $\left(\omega_{f_{x}}^{*}=0.6\right)$, there is a mode of maximum instability, where the square normalized growth rate $\gamma^{2}$ increases with $k^{* 2}$ increases through the range $0<k^{* 2}<k_{\max }^{* 2}$ (at $k_{\max }^{* 2}$ the square normalized growth rate arrives to the maximum instability, and when $k^{* 2}>k_{\max }^{* 2}$ the square normalized growth rate $\gamma^{2}$ starts to decreases as $k^{* 2}$ increases and then goes to the complete stable at $k_{c}^{* 2}$ ( $k_{c}^{* 2}$ is the critical value for stability, at this point the square normalized growth rate goes to zero). This means that the horizontal component of mag- 
netic field has a crucial capability to suppress the instability that satisfies for the large wave number (short wavelength), while the vertical component of magnetic field has this strength just for the very short wave number (large wavelength). Also, the considerable model is more stability in the presence of both horizontal and vertical components of the magnetic field $\left(\omega_{f_{x}}^{*}=\omega_{f_{z}}^{*}=0.6\right)$. This case $\left(\omega_{f_{x}}^{*}, \omega_{f_{z}}^{*}\right)$ shows in Figure 4, where the magnitudes of $\gamma^{2}$ decrease with increasing of both horizontal and vertical components of magnetic field

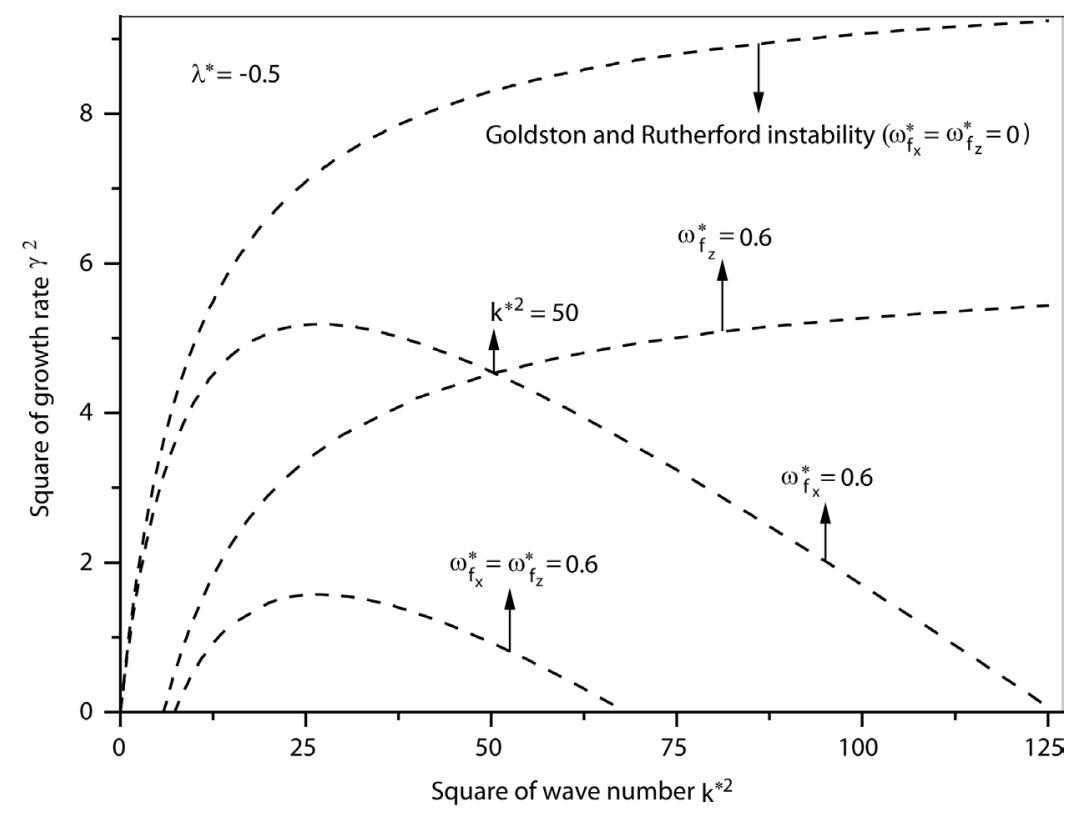

Figure 3. The square normalized growth rate $\left(\gamma^{2}\right)$ against the square normalized wave number $k^{* 2}$ at $\lambda^{*}=-0.5$ in the presence of $\omega_{f_{x}}^{*}=0.6, \omega_{f_{z}}^{*}=0.6$ and $\omega_{f_{x}}^{*}=\omega_{f_{z}}^{*}=0,0.6$.

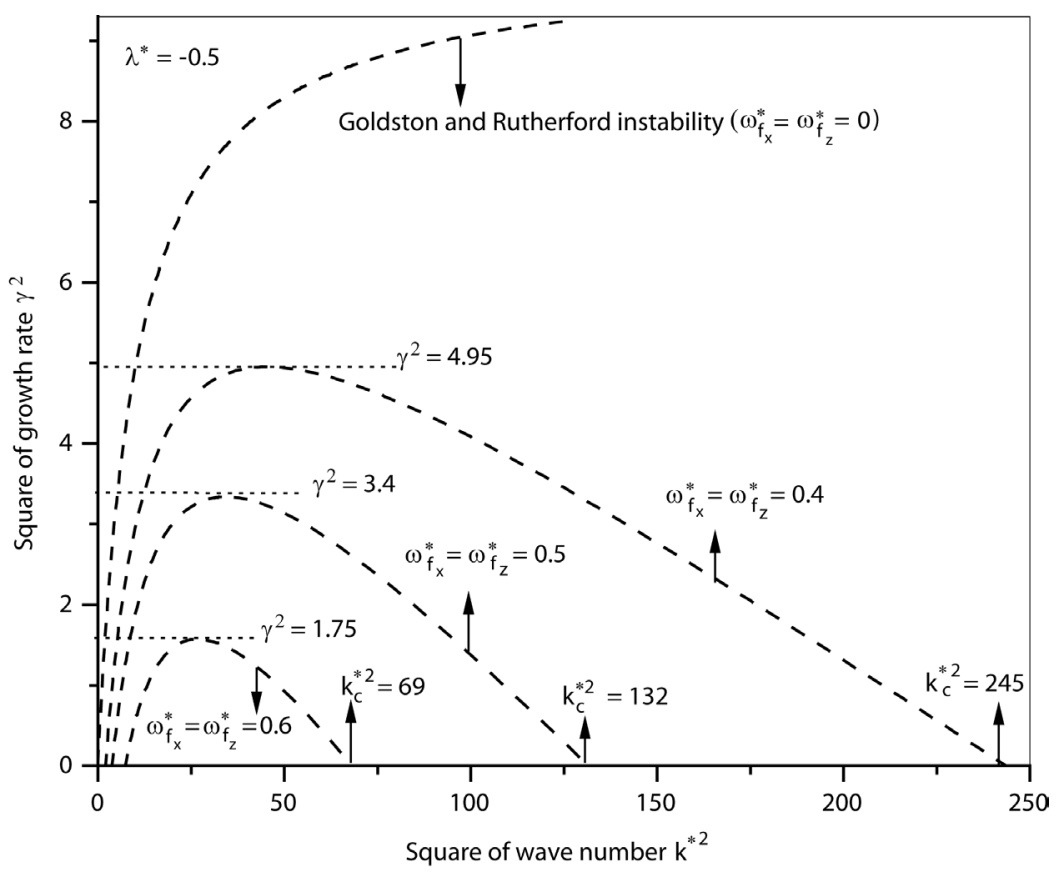

Figure 4. The square normalized growth rate $\left(\gamma^{2}\right)$ against the square normalized wave number $k^{* 2}$ at $\lambda^{*}=-0.5$ for different values of the magnetic field $\omega_{f_{x}}^{*}=\omega_{f_{z}}^{*}=0,0.4,0.5,0.6$. 
$\left(\omega_{f_{x}}^{*}=\omega_{f_{z}}^{*}=0.4,0.5,0.6\right)$ that are less than their counterpart at $\omega_{f_{x}}^{*}=\omega_{f_{z}}^{*}=0$ (Goldston and Rutherford results). Figure 5(a) and Figure 5(b) show the role of magnetic field $\left(\omega_{f_{x}}^{*}=\omega_{f_{z}}^{*}=0.4,0.5,0.6\right)$ when $\lambda^{*}$ have

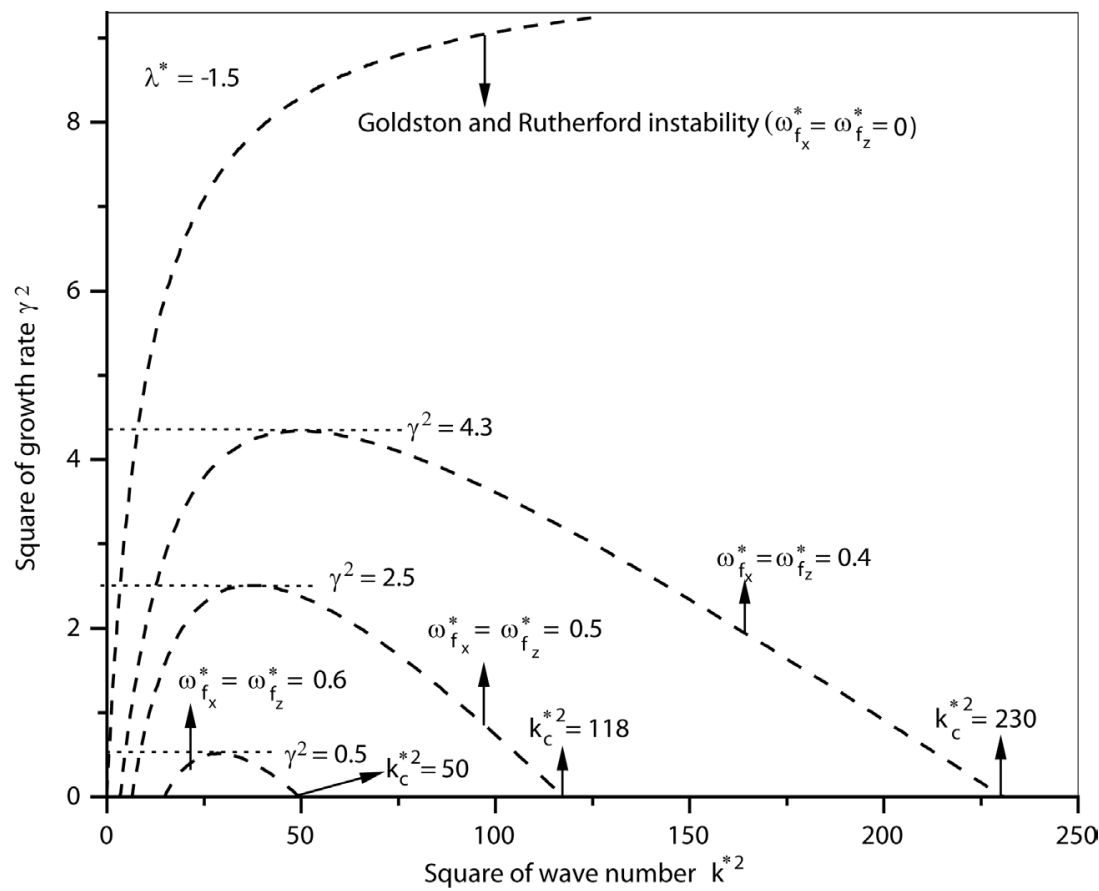

(a)

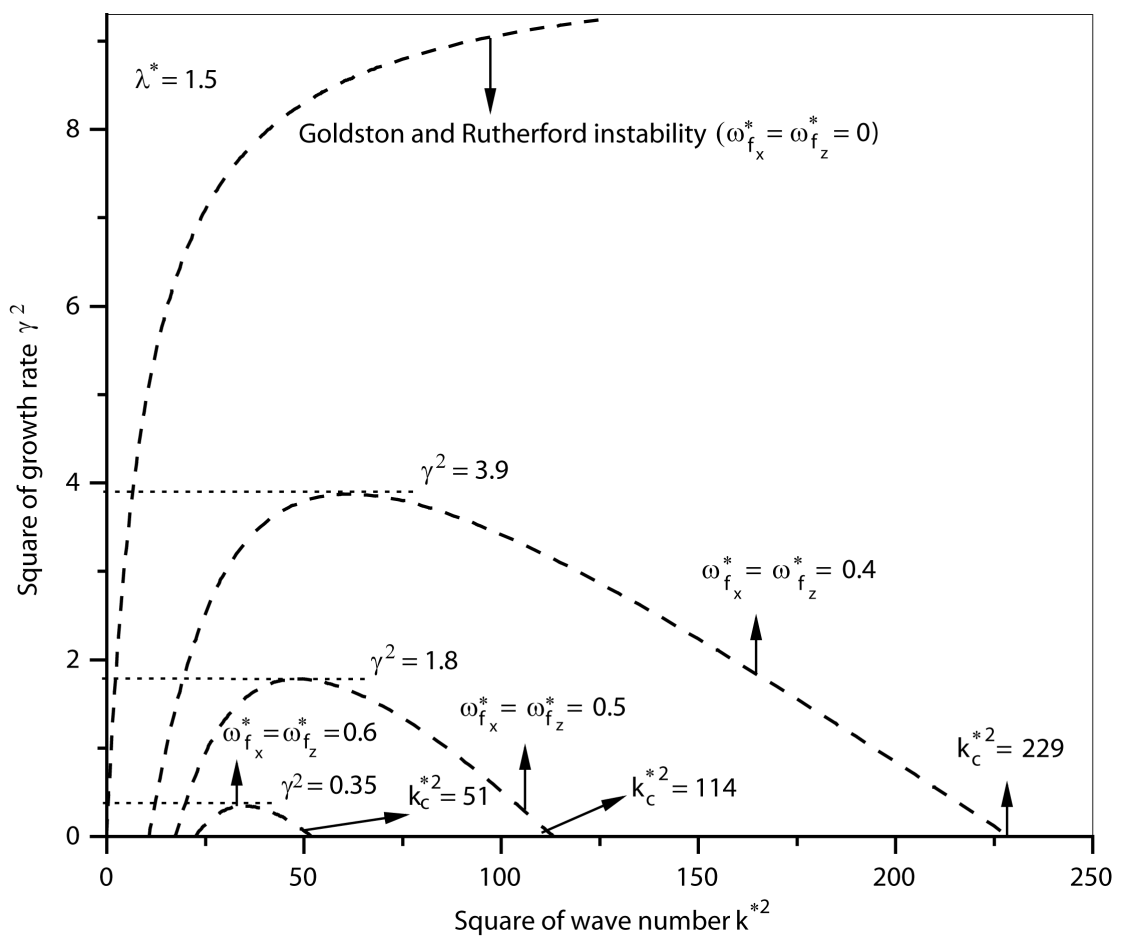

(b)

Figure 5. The square normalized growth rate $\left(\gamma^{2}\right)$ against the square normalized wave number $k^{* 2}$ for different values of the magnetic field $\omega_{f_{x}}^{*}=\omega_{f_{z}}^{*}=0,0.4,0.5,0.6$. (a) at $\lambda^{*}=-1.5$ (b) at $\lambda^{*}=1.5$. 
different values than $\lambda^{*}=-0.5$ (at $\lambda^{*}=-1.5,1.5$, respectively). In Figure 5(a), one can see that, the maximum instability (maximum square of growth rate $\left.\gamma^{2}\right)$ at $\left(\omega_{f_{x}}^{*}=\omega_{f_{z}}^{*}=0.4,0.5,0.6\right)$ in Figure 5(a), respectively, are $\left(\gamma^{2}=4.2,2.5,0.5\right)$, they are less than their counterpart in Figure $4\left(\gamma^{2}=4.95,3.4,1.17\right)$. Also, the critical point for stability in Figure 5(a), respectively, are $\left(k_{c}^{* 2}=230,118,50\right)$, they are less than their counterpart in Figure $4\left(k_{c}^{* 2}=245,132,69\right)$. This implies that the magnitudes of $\gamma^{2}$ in Figure 5(a) at $\lambda^{*}=-1.5$ are less than their counterpart in Figure 4 at $\lambda^{*}=-1.5$. The same phenomenon holds in Figure 5 (b) but at $\lambda^{*}=1.5$, where both the maximum instability and the critical point for stability in Figure 5(b) are less than their counterpart in Figure 4 at $\lambda^{*}=-0.5$, which also indicates that, the magnitudes of $\gamma^{2}$ at $\lambda^{*}=-1.5$ are less than their counterpart at $\lambda^{*}=-0.5$.

\section{Conclusions}

Finally, we have investigated the effect of magnetic field in both horizontal and vertical direction on the Rayleigh-Taylor instability of stratified incompressible plasmas layer. We can summarize the results as follows:

(i) In the presence of horizontal magnetic field only or vertical magnetic field only the growth rate depends on $\left(h^{*}=\frac{h}{L}\right)$ of plasma layer and $\lambda^{*}$ takes one value $\left(\lambda^{*}=-0.5\right)$. While, in the presence of both horizontal and vertical magnetic field, the square growth rate (Equation (32)) depends on the dimensionless thickness $\left(h^{*}=\frac{h}{L}\right)$ and also depends on the parameter $\lambda^{*}=\lambda L_{D}$.

(ii) The constant $\lambda^{*}=\lambda L_{D}$ plays an important stabilizing role in the growth rate, where the maximum instability happens at $\lambda^{*}=-0.5$ and to get more stability model, the values $\lambda^{*}$ must be different than $\lambda^{*}=-0.5$ (i.e. $-0.5<\lambda^{*}$ or $\lambda^{*}<-0.5$ ). In other words, in studying the combined effect of horizontal and vertical magnetic field on RTI of stratified plasma, the system will be more stable when we accept that $\lambda^{*}$ is different than -0.5 (i.e. $\lambda^{*} \neq-0.5$ ).

(iii) Our model is more stable than those considered in previous study (horizontal magnetic field only or vertical magnetic field only). This divergence ascribes to the stabilizing role that the magnetic field plays on RTI problem in the presence of both horizontal and vertical components, where the presence of both these parameters dissipates the energy of any disturbance and thereby the system becomes more stability.

\section{References}

[1] Rayleigh, L. (1882) Investigation of the Character of the Equilibrium of an Incompressible Heavy Fluid of Variable Density. Proceedings of the London Mathematical Society, 14, 170-177.

[2] Taylor, G.I. (1950) The Instability of Liquid Surfaces When Accelerated in a Direction Perpendicular to Their Planes. Proceedings of the Royal Society of London. Series A, Mathematical and Physical Sciences, 201, 192-196.

[3] Sanz, J. (1994) Self-Consistent Analytical Model of the Rayleigh-Taylor Instability in Inertial Confinement Fusion. Physical Review Letters, 73, 2700-2703. http://dx.doi.org/10.1103/PhysRevLett.73.2700

[4] Lindl, J.D. (1995) Development of the Indirect-Drive Approach to Inertial Confinement Fusion and the Target Physics Basis for Ignition and Gain. Physics of Plasmas, 2, 3933. http://dx.doi.org/10.1063/1.871025

[5] Cabot, W.H. and Cook, A.W. (2006) Reynolds Number Effects on Rayleigh-Taylor Instability with Possible Implications for Type-Ia Supernovae. Nature Physics, 2, 562-568.

[6] Timmes, F.X. and Woosley, S.E. (1992) The Conductive Propagation of Nuclear Flames. I - Degenerate C + O and O + NE + MG White Dwarfs. Astrophysical Journal, 396, 649-667. http://dx.doi.org/10.1086/171746

[7] Blinnikov, S. and Sorokina, E. (2004) Type Ia Supernova Models: Latest Developments. Astrophysics and Space Science, 290, 13-28. http://dx.doi.org/10.1023/B:ASTR.0000022161.03559.42

[8] Chen, F.F. (1974) Introduction to Plasma Physics. Plenum, New York.

[9] Goldston, R.J. and Rutherford, P.H. (1997) Introduction to Plasma Physics. Institute of Physics, London.

[10] Ariel, P.D. (1971) Rayleigh-Taylor Instability of Compressible Fluids in the Presence of a Vertical Magnetic Field. Applied Scientific Research, 24, 294-304.

[11] Bhatia, P.K. (1974) Rayleigh-Taylor Instability of a Viscous Compressible Plasma of Variable Density. Astrophysics and Space Science, 26, 319-325. http://dx.doi.org/10.1007/BF00645614 
[12] Shivamoggi, B.k. (1981) Rayleigh-Taylor Instability of Compressible Plasma in a Vertical Magnetic Field. Astrophysics and Space Science, 79, 3-9. http://dx.doi.org/10.1007/BF00655900

[13] Ariel, P.D. (1991) Rayleigh-Taylor Instability of a Hall Plasma with Arbitrary Density Gradient. Astrophysics and Space Science, 184, 205-219.

[14] Ali, A. and Bhatia P.K. (1993) Rayleigh-Taylor Instability of a Stratified Hall Plasma in Two-Dimensional Horizontal Magnetic Field. Physica Scripta, 47, 567-570. http://dx.doi.org/10.1088/0031-8949/47/4/016

[15] Khan, A. and Bhatia, P.K. (1993) Rayleigh-Taylor Instability of a Finitely Conducting Partially Ionized Hall Plasma. Physica Scripta, 48, 607-611. http://dx.doi.org/10.1088/0031-8949/48/5/017

[16] Wu, Z., Zhang, W., Li, D. and Yang. W. (2004) Effect of Magnetic Field and Equilibrium Flow on Rayleigh-Taylor Instability. Chinese Physics Letters, 21, 2001-2004.

[17] Cao, J.T., Ren, H.J., Wu, Z.W. and Chu, P.K. (2008) Quantum Effects on Rayleigh-Taylor Instability in Magnetized Plasma. Physics of Plasmas, 15, 012110. http://dx.doi.org/10.1063/1.2833588

[18] Hoshoudy, G.A. (2010) Quantum Effects on Rayleigh-Taylor Instability of Incompressible Plasma in a Vertical Magnetic Field. Chinese Physics Letters, 27, 125201. http://dx.doi.org/10.1088/0256-307X/27/12/125201

[19] Hoshoudy, G.H. (2011) Rayleigh-Taylor Instability in Quantum Magnetized Viscous Plasma. Plasma Physics Reports, 37, 775-784. http://dx.doi.org/10.1134/S1063780X11080046

[20] Yang, B.L., Wang, L.F., Ye, W.H. and Xue, C. (2011) Magnetic Field Gradient Effects on Rayleigh-Taylor Instability with Continuous Magnetic Field and Density Profiles. Physics of Plasmas, 18, 072111. http://dx.doi.org/10.1063/1.3609773

[21] Wang, L.F., Yang, B.L., Ye, W.H. and He, X.T. (2012) Stabilization of the Rayleigh-Taylor Instability in Quantum Magnetized Plasmas. Physics of Plasmas, 19, 072704. http://dx.doi.org/10.1063/1.4737162 
Scientific Research Publishing (SCIRP) is one of the largest Open Access journal publishers. It is currently publishing more than 200 open access, online, peer-reviewed journals covering a wide range of academic disciplines. SCIRP serves the worldwide academic communities and contributes to the progress and application of science with its publication.

Other selected journals from SCIRP are listed as below. Submit your manuscript to us via either submit@scirp.org or Online Submission Portal.
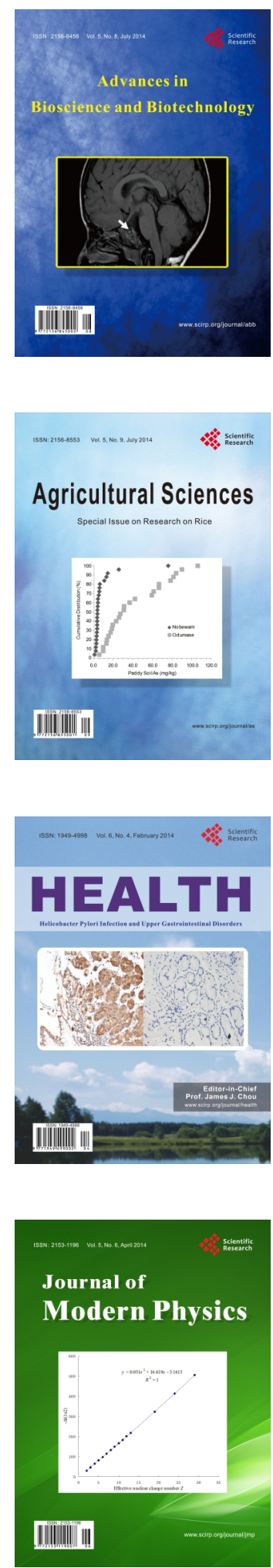
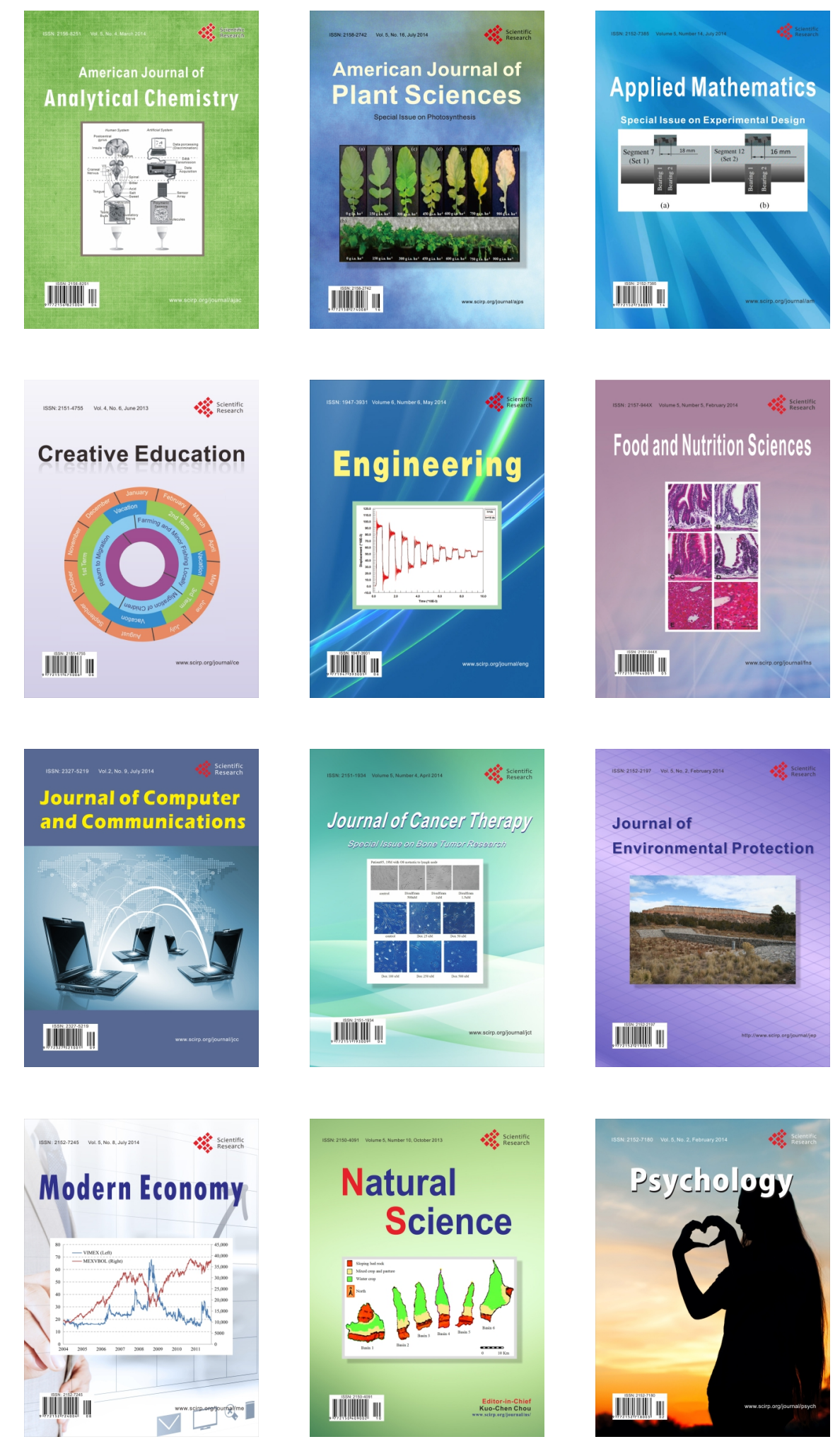